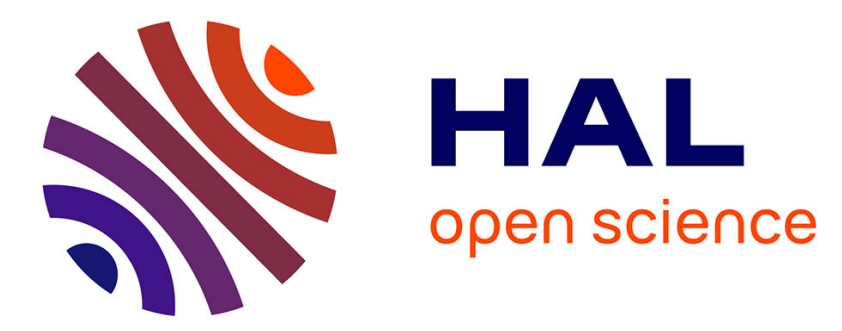

\title{
PAVEMENT CRACKING DETECTION USING AN ANISOTROPY MEASUREMENT
}

Tien Sy Nguyen, Stéphane Begot, Florent Duculty, Jean-Christophe Bardet, Manuel Avila

\section{- To cite this version:}

Tien Sy Nguyen, Stéphane Begot, Florent Duculty, Jean-Christophe Bardet, Manuel Avila. PAVEMENT CRACKING DETECTION USING AN ANISOTROPY MEASUREMENT. 11ème IASTED International Conference on Computer Graphics and Imaging (CGIM), Feb 2010, Innsbruck, Austria. hal-00608266

\section{HAL Id: hal-00608266 https://hal.science/hal-00608266}

Submitted on 12 Jul 2011

HAL is a multi-disciplinary open access archive for the deposit and dissemination of scientific research documents, whether they are published or not. The documents may come from teaching and research institutions in France or abroad, or from public or private research centers.
L'archive ouverte pluridisciplinaire HAL, est destinée au dépôt et à la diffusion de documents scientifiques de niveau recherche, publiés ou non, émanant des établissements d'enseignement et de recherche français ou étrangers, des laboratoires publics ou privés. 


\title{
PAVEMENT CRACKING DETECTION USING AN ANISOTROPY MEASUREMENT
}

\author{
Tien Sy Nguyen, Stephane Begot, Florent Duculty, Jean-Christophe Bardet, Manuel Avila \\ PRISME Institute \\ University of Orléans, IUT de l'Indre \\ 2 av; F. Mitterrand \\ 36000 CHATEAUROUX \\ FRANCE \\ Tien-Sy.Nguyen@etu.univ-orleans.fr
}

\begin{abstract}
Automatic pavement cracking detection is a part of road maintenance and rehabilitation strategies. Cracks detection is one of the main features used by road authorities to manage efficiently its networks.

Different systems are available to perform road analysis. We give a short description of some of them. Apparatus which was used to provide our images is described with more details.

Road surface is made using randomly organized aggregates which can have different sizes. Scanned pictures of theses surfaces appear as random distribution of a reduced set of gray levels. Automatic crack detection is a difficult task due to the noisy pavement surface.

In this paper, we introduce a measure of anisotropy for the characterization of cracks. The basic idea of this method is to detect the variation of features by considering different orientations. Noise variation and defect properties can be take into account by our method. Comparative results of anisotropy method with threshold method and 2D wavelet transform method are presented
\end{abstract}

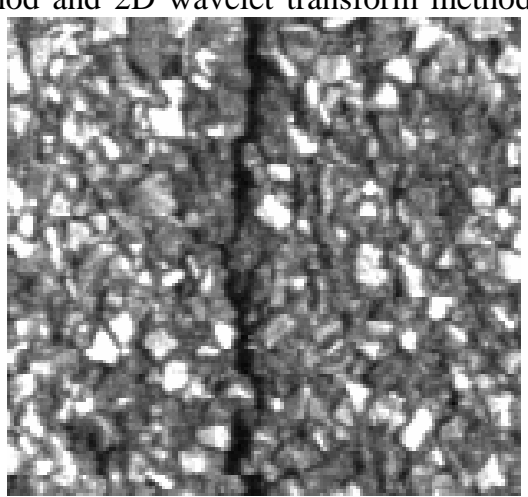

(a)

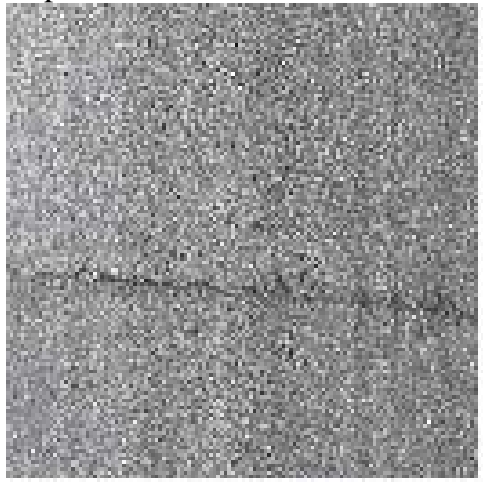

(b) to illustrate benefits of anisotropy. We show that this method can be used to detect others types of defects, such as joints.

\section{KEY WORDS}

crack detection, image analysis, road classification

\section{Introduction}

To efficiently manage the road network, road authorities need accurate and up-to-date information on the road condition. Defect information is vital to keep wellmaintained road networks. Nowadays, in France, thousands of kilometers of roads are inspected each year. Because huge amount of image data must be inspected, boringness of inspection task, and in addition easy-tomake-mistake task, the automatic pavement cracking detection is highly required [1].

There are several types of defects on road, some examples are shown in Figure 1.

Fig. 1. Some examples of road defects: longitudinal crack (a), transversal crack (b), alligator crack (c).

Cracks are the most frequent defect type. Road managers need to detect and to fix these defaults before they turn into serious problems such as pot-holes and pop-outs.

Pavement cracking detection is not a "simple" edge detection problem or a texture analysis problem due to the nature of these images. Various pavement textures can be encountered on pavement surface images. Secondly, many road surfaces are "strong textures" [2]. Cracks can be thinner than aggregate size and space between them. Distribution of aggregates in pavement is strongly random due to variation of their size and their random organization. There could be a lot of noise in the images. Light condition and surface of pavement can provide lot of variation.

Consequently, it is hard to distinguish a crack with aggregate distribution. Find out "homogenous" features of road texture to detect pavement cracks is a very difficult task.

General approaches of defect detection in textured surfaces try to find a "homogeneous" feature of "defect- 
free" textures and compare the differences caused by defects presence [3]. Several methods for defects detection in textured surface have been discussed in [4], but most of these methods are for defects detection on uniform or nearly uniform texture. In these studies, two important features were used. These features are orientation and pattern frequency. None of these methods have been successful in pavement crack detection.

Most of existing methods of pavement crack detection are based on feature of crack, not on feature of "free defect" road surface. A scheme for crack detection method is usually divided in four steps: Pre-processing, Image segmentation, Post-processing and Crack extraction. A short summary of different methods used in each step is shown in Table 1.

Unexpected effect of acquisition task such as illumination condition (shadows), humidity or pavement change is reduced by pre-processing step.

The main task of crack detection is segmentation step. Two main assumptions are made about crack features. Local extremum or darker regions are considered as crack pixels. For example, Subirats et al. [2][5], segment road images by thresholding through different scales using a 2D continuous wavelet transform.

Post-processing step is used to reduce noise and to connect small segments of cracks.
The last step, the cracks extraction is to find out presence of cracks and locate them on images.

\begin{tabular}{|l|l|}
\hline Pre-processing & $\begin{array}{l}\text { Contrast stretching [1, 7] } \\
\text { Equalization histogram [3,6] }\end{array}$ \\
\hline Road image segmentation & $\begin{array}{l}\text { Fixed threshold[1, 8-11] } \\
\text { Fuzzy entropy threshold [6, 7] } \\
\text { Wavelet transform and threshold } \\
\text { on the space of coefficient [2,5] }\end{array}$ \\
\hline Post-processing & $\begin{array}{l}\text { Morphology operation [1, 6, 12] } \\
\text { Connectivity Searching [6, 13-15] }\end{array}$ \\
\hline Crack extraction & $\begin{array}{l}\text { Projection [1, 2, 6, 16] } \\
\text { Hough transform [2, 6] } \\
\text { Neural Networks [2, 13] }\end{array}$ \\
\hline
\end{tabular}

Table 1: Summary of methods for crack detection on road surface images.

Most of these existing methods, only use local feature of crack to segment images. Crack pixels are considered as darker pixels or as local extrema, but connectivity and orientation of crack were not considered in the segmentation process. In fact, due to nature of road textures, many crack-free pixels have a grey level as low as crack pixel. Consequently, result of segmentation could contain noises (example in Figure 2).

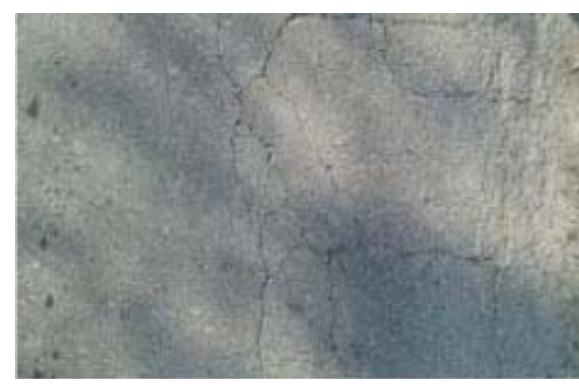

(a)

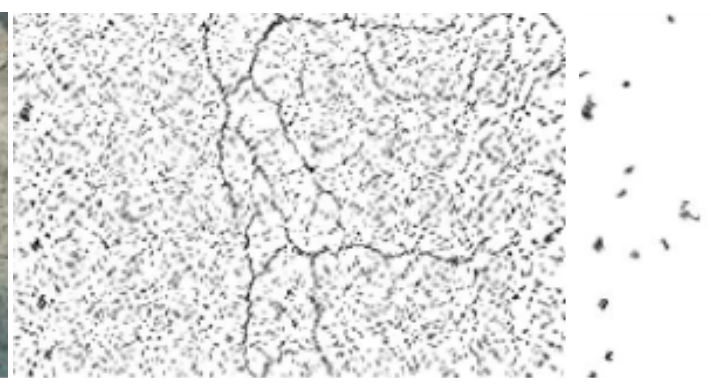

(b)

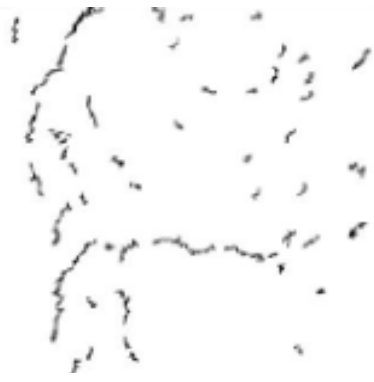

(c)

Fig. 2. Road crack detection by Subirat [2]: inspected image (a), crack candidate pixel (b), result after post-processing (c).

Geometrical characteristics of cracks have been taken into account in the post-processing step to reduce noise and to connect crack pixels. But this task of discrimination between the noisy pixels and crack pixels is not an easy task. In [3], Oliveira \& al use a Bayesian classifier applied on small windows of the image. Features used are mean and standard deviation of pixel intensities. Sample images, shown, seem to be captured in optimal condition. There is a few variation of pixel intensities. It is not true in case of dynamic acquisition with a line scan camera on high performance apparatus [17].

\section{Outline on actual system for road crack inspection}

\subsection{On-road visual defect detection}

First methods of road crack detection are on-road visual detection. Technicians travel in a vehicle at speed of 5 to 10 kilometers per hour. Driver watches the road and when a crack is found, passenger records the cracks onto a dedicated board acquisition device. This method is slow and unsafe not only for road monitoring staff but also for road users.

To overcome limitations of visual inspection, the idea is: Whether "to bring road to office"- Road images are firstly captured by a high speed acquisition device, and then, the inspection process is done offline by inspectors viewing captured images. Or, the device must be able to detect automatically road defects in real time and at high speed. Some such devices are CSIRO's RoadCrack vehicle [18], Roadware's ARAN system [19]. Unfortunately, because of the commercial nature of these systems, hardware and software used have not been published. Besides, by difference of road pavement texture, tests made in French road network show that both of ARAN and RoadCrack systems do not satisfy the requirements for French road maintenance. Road maintenance in France requires information about cracks as small as $1 \mathrm{~mm}$ wide. 


\subsection{AMAC ${ }^{\circledR}$ System for Road Analysis in France} Vectra have developed system AMAC ${ }^{\circledR}$ [17] - (Figure 3(a)) for road characterization in France. One of its functions is acquisition of road images in high resolution and independently of lighting conditions. Data acquisition is performed with two line scan cameras mounted on the

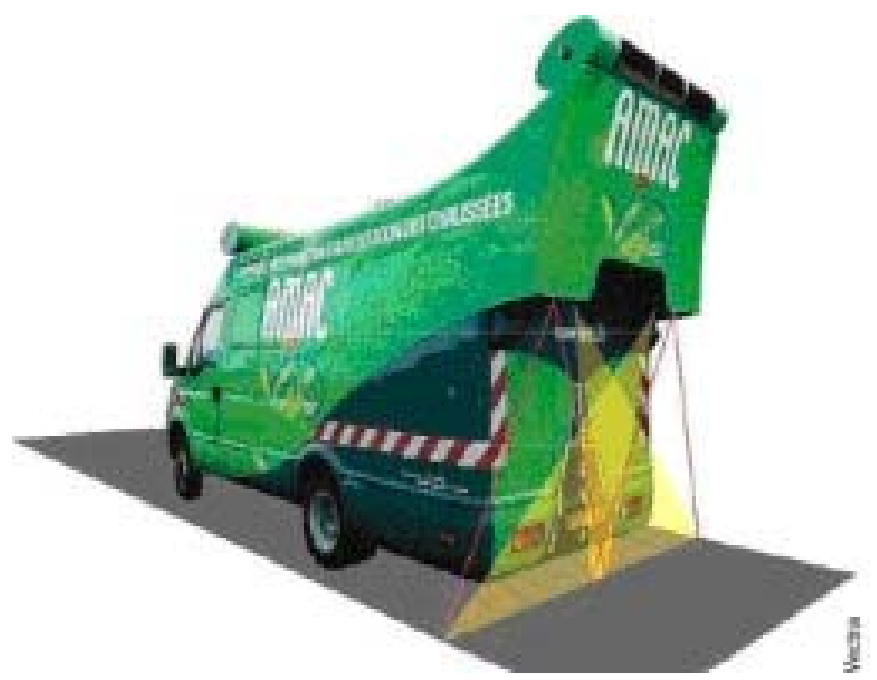

(a) vehicle with an angle of approximately 30 degrees, road surface is illuminated by two laser illuminators (Figure 3(b)), thus the time of day, varying light conditions and shadows over the road have almost no effect on the quality of acquired data [1].

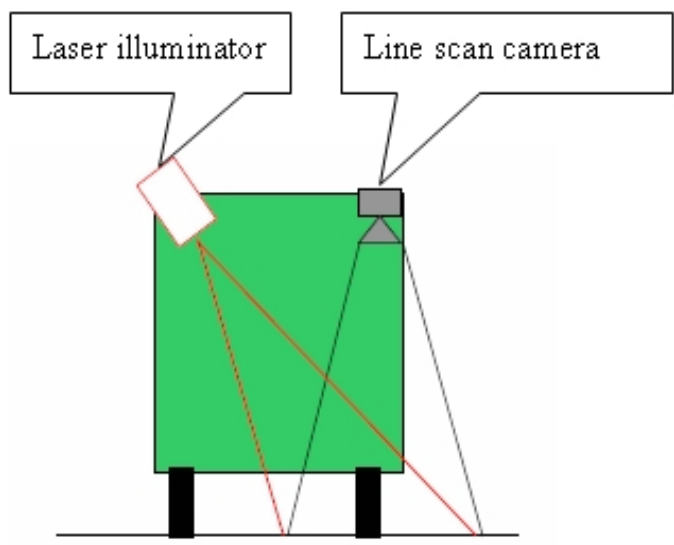

(b)

Figure 3. AMAC® apparatus for road analysis (a), image acquisition system used on AMAC® (b)

The vehicle works at an average speed of 80 kilometers per hour (to meet requirement of speed limitations in highway and motorway). The resolution of acquired image is $1 \mathrm{~mm}$ per pixel; the size of road captured by each image is $3.9 \mathrm{~m}$ width and $4 \mathrm{~m}$ long, corresponding to the average size of $4096 \times 4096$ pixels, 8 bits grey level image. Each second, about 5 images of this large size must be stored, it means that about one terabyte of images are stored in each session of acquisition and then must be analyzed by inspector. Actually, inspection process is done manually by operators viewing acquired images on screens. Each operator is able to analyze 15 to $20 \mathrm{~km}$ of road per day.

Because huge amount of image data must be inspected, the boringness of inspection task, and in addition, the easy-to-make-mistake task, automatic or semi-automatic detection of cracks on road images is highly required.

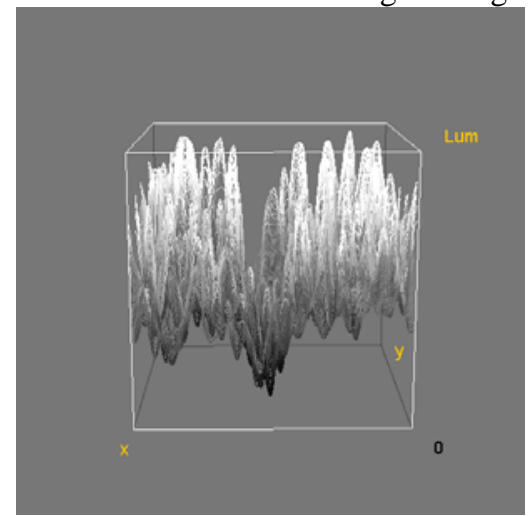

(a)

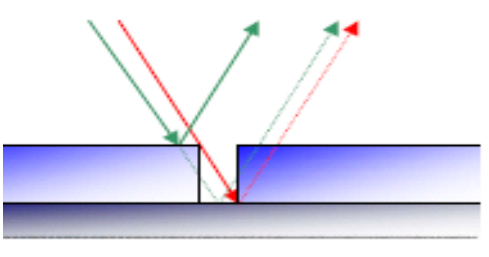

(b)

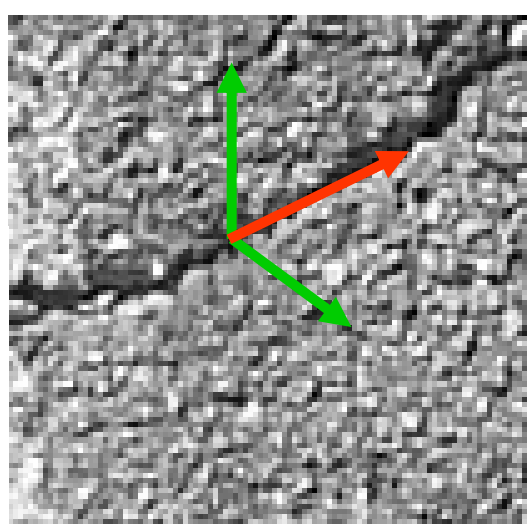

(c)

Fig. 4. Analysis of a crack, 3D profile (a), illumination model on a crack (b), continuity on a given orientation (c). 


\subsection{Definition of Conditional Texture Anisotropy}

Now, we introduce an algorithm to segment crack images. This method take into account the three characteristics (3.1): gray intensities, connectivity and orientation of cracks during segmentation step.

Conditional Texture Anisotropy (CTA) was introduced by F. Roli in [20] The main idea is to find out a measure which has a different value in one direction (along the orientation of crack) between other directions. In [20], CTA was defined as:

$$
\operatorname{CTA}\left(X^{l}\right)=\frac{\max _{j}\left\{p\left(x_{j}^{l} / l \in w_{1}\right)\right\}-\min _{j}\left\{p\left(x_{j}^{l} / l \in w_{1}\right)\right\}}{\max _{j}\left\{p\left(x_{j}^{l} / l \in w_{1}\right)\right\}}
$$

With vector $X^{l}=\left(x_{1}^{l}, x_{2}^{l}, \ldots x_{m}^{l}\right)$ composed of sub-vector $x_{j}^{l}=\left(\right.$ feature $\_1_{j}^{l}$, feature $\_2_{j}^{l}, \ldots$ feature $\left.\_k_{j}^{l}\right)$ representing $k$ different features of $l$ pixel along different $j$ orientations (e.g. $0^{\circ}, 45^{\circ}, 90^{\circ}$ and $135^{\circ}$ ).

According to pattern recognition theory, function $p\left(x_{j}^{l} / l \in w_{1}\right)$ ( $w_{1}$ being the class of defect-free pixels) can be computed with parametric or non parametric techniques [11].

As described in [20], CTA takes values close to 0 for freedefect region. In this case, feature values extracted according to each orientation are close together. Around defects, CTA will take values close to 1. For example, along crack orientation, feature values will be very different from others directions.

This measure is said to be 'conditional' because it takes into account the kind of texture.

In [18], F. Roli gives a CTA example for crack detection on granite slab images affected by crack. Four texture features were computed using the Fourier power spectrum. Nonetheless, the formula for calculating CTA was not explained.

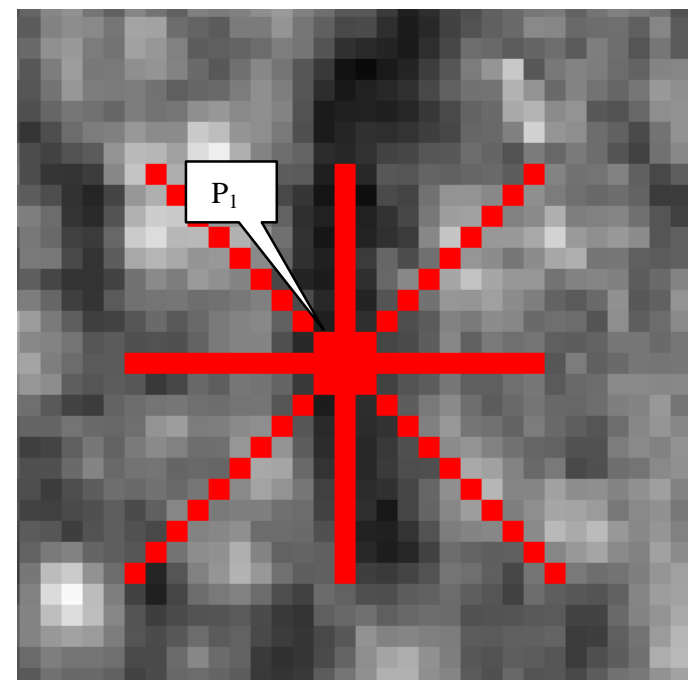

zoom

\subsection{Crack detection on road surface by CTA}

According to cracks characteristics on road surface images, we have chosen mean and standard deviation as two features for calculating CTA. The method of calculation is presented as follows:

$2 * d+1$ is the length of a line, along which we calculate each feature. $d$ should be chosen as $2 * d+1>>$ aggregate size to satisfy assumptions made in part 3.1.

Four directions $0^{\circ}, 45^{\circ}, 90^{\circ}$ and $135^{\circ}$ are considered.

mean $m_{j}^{l}$ and standard deviation $\sigma_{j}^{l}$, of $2 * \mathrm{~d}+1$ pixels along considered direction $j$, are calculated.

Feature vector of pixel $l$ along $j$ orientation is

$$
x_{j}^{l}=\left(m_{j}^{l}, \sigma_{j}^{l}\right)
$$

To evaluate the pre-assumed probability $p\left(x_{j}^{l} / l \in w_{1}\right)$ of a pixel $l$ to be "a defect-free pixel according to orientation j”, we calculate :

$$
p\left(x_{j}^{l} / l \in w_{1}\right)=\frac{D-\Delta_{j}}{D}
$$

with $D=\sqrt{m^{2}+\sigma^{2}}$ and $\Delta_{j}=\sqrt{\left(m-m_{j}^{l}\right)^{2}+\left(\sigma-\sigma_{j}^{l}\right)^{2}}$

$\mathrm{m}$ and $\sigma$ are calculated on whole the image. These formula can be compared to a normalized distance which is close to 1 for defect-free pixel. Mean and standard deviation according to all directions are near "global" values. In case of crack pixel, distance $\Delta_{j}$ along the orientation of crack is higher and value decrease towards zero. CTA is then calculated as in equation (1). Remember that this is a pre-assumed probability value used for calculating CTA. We do not detect the crack by using this probability but by calculating CTA. The information which plays the most important role for crack detection is the distribution of mean and standard deviation values according to all orientations.

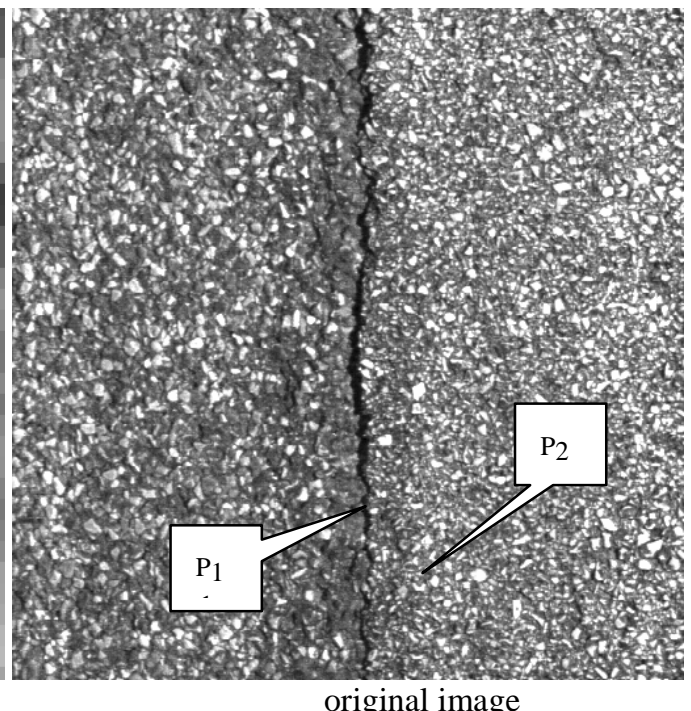

original image 


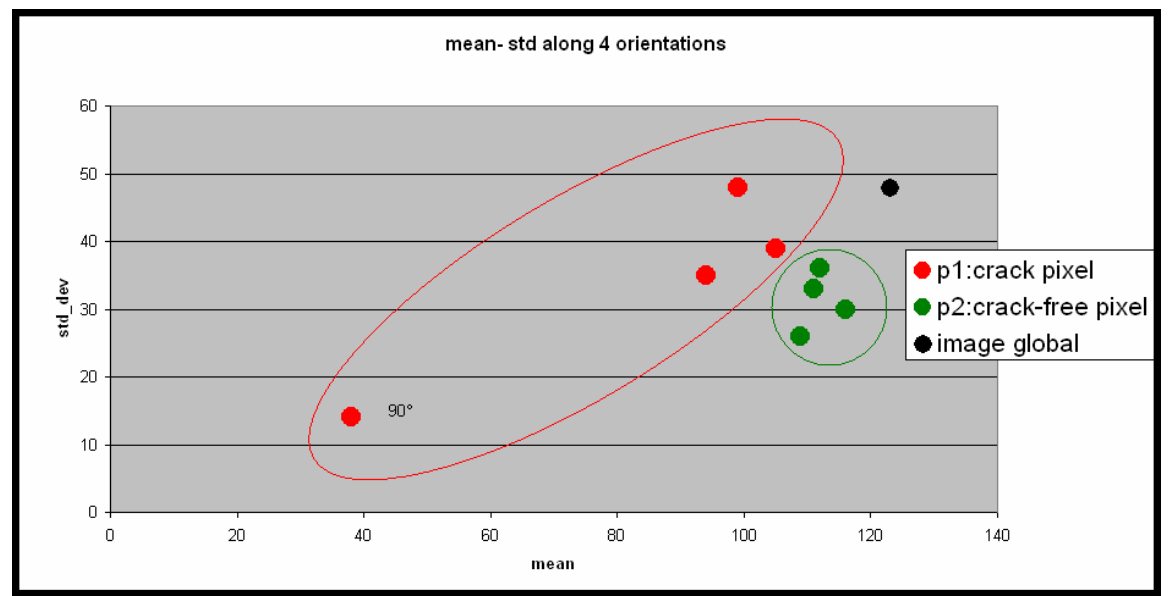

Fig. 5. Example of CTA for $p_{1}$ pixel, $d=10$. Distribution of $m$ and $\sigma$ calculated on $p_{1}$ and $p_{2}$ along 4 orientations.

Figure 5 illustrates a calculation sample of CTA on these two pixels, $\mathrm{P}_{1}$ (a crack pixel) and $\mathrm{P}_{2}$ (a free defect pixel). Values of mean and standard deviation $\left(m_{j}^{l}, \sigma_{j}^{l}\right)$ along 4 orientations are:

(99.1, 47.5), (93.7, 34.5), (37.9, 14.3) and (104.7, 39.4). With $(123.5,48.1)$ global values, value of CTA of $\mathrm{P}_{1}$ is 0.64 .

With pixel $\mathrm{P}_{2}$, values of $\left(m_{j}^{l}, \sigma_{j}^{l}\right)$ along 4 orientations are :

(111.9, 36.0), (108.9, 25.8), (110.5, 33.4), (116.0, 29.6).

The value of CTA of $\mathrm{P}_{2}$ is $\mathbf{0 , 0 8}$.

The distribution of CTA on a road surface image was supposed to be a Gaussian distribution (this hypothesis was verified in our experiment of road image analysis).
After calculating all values of CTA in an inspected image, for each inspected pixel $l$, the Mahalanobis distance is computed as:

$$
d_{M}(l)=\frac{\left(C T A\left(X^{l}\right)-M_{C T A}\right)^{2}}{\sigma_{C T A}^{2}}
$$

Where $\mathrm{M}_{\mathrm{CTA}}$ and $\sigma_{\text {CTA }}^{2}$ are mean and variance of CTA distribution of inspected image.

As we see in figure 6 (b) and (e), values of crack pixel with Mahalanobis distance images are very high and contrast with others free defect pixels of images. Crack pixels are easily detected (Figure 6 (c), (f)) by a threshold from these distance images.

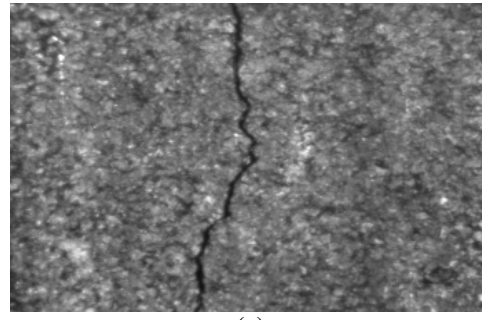

(a)

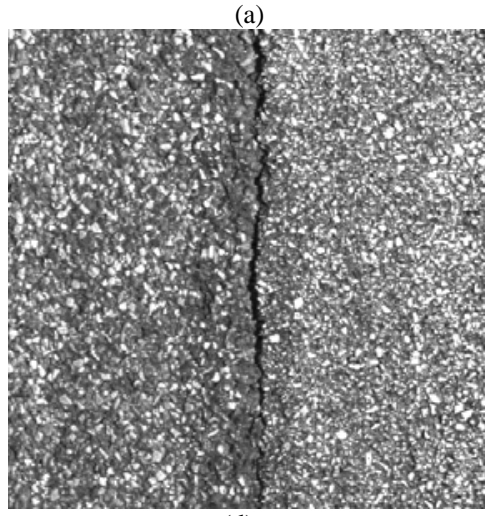

(d)

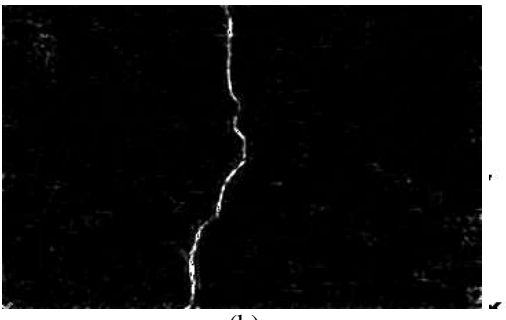

(b)

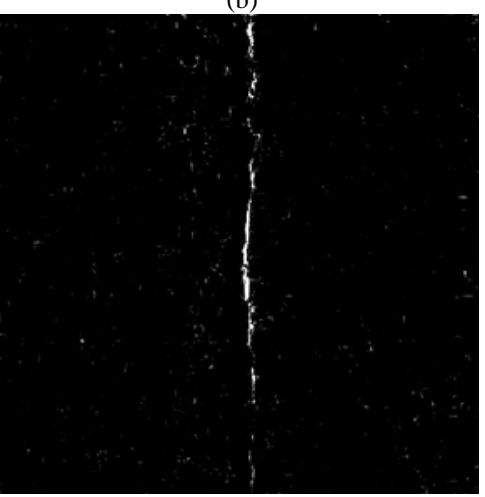

(e)

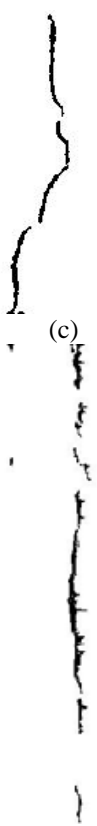

(f)

Fig. 6. Example of detection results. original images $(a, d)$, Mahalanobis distance results $(b, e)$ . crack detection results $(c, f)$. 


\section{Experimental Results}

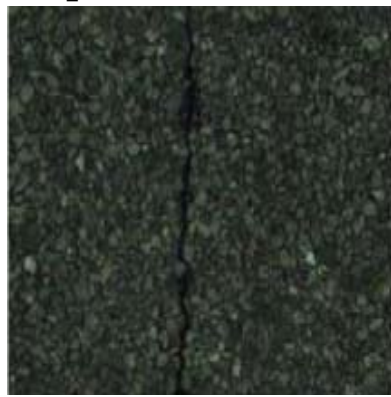

(a)

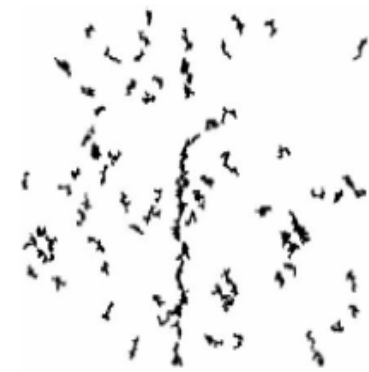

(b)

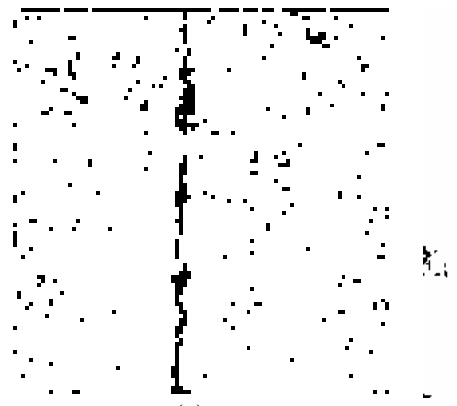

(c)

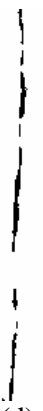

(d)

Fig. 7. Anisotropy vs. Subirat's 2D wavelet transform method: inspected image (a), Subirat's method (b), threshold method (c), our CTA method (d).

We see comparative results of our method with threshold method [1] and Subirat's method [2]. The 2D wavelet transform raises the crack but it increases also noises because continuity was not considered (Figure 7 (b)). In the other hand, our method only raises the continuous crack pixels. The threshold method (Figure 7 (c)) has limitations because it cannot differentiate a dark pixel of defect-free texture from a crack pixel.

In our database, images should contain more than one kind of defect (joint, crack, pothole, ...). In this paper, we only test crack detection, so we have chosen images containing only cracks.

Table 2. shows results of our method tested on about 540 images, 235 crack images and 307 defect-free images. These images were selected randomly from our image databases which were already labeled by operators. Our experiments show that this method provides a very good detection of longitudinal and transversal cracks. Our method can detect cracks as small as $2 \mathrm{~mm}(1 \mathrm{~mm}=1$ pixel), even the "well" continuous cracks as small as $1 \mathrm{~mm}$ can be detected (Figure 6 (a)).

\begin{tabular}{|l|l|l|l|l|}
\hline Image type & $\begin{array}{l}\text { Number } \\
\text { of } \\
\text { images }\end{array}$ & Errors & $\begin{array}{l}\text { Percentage } \\
\text { of images } \\
\text { detected as } \\
\text { crack image }\end{array}$ & $\begin{array}{l}\text { Percentage of } \\
\text { images } \\
\text { detected as no- } \\
\text { crack image }\end{array}$ \\
\hline Crack & 235 & 15 & $93,6 \%$ & $6,4 \%$ \\
\hline Defect-free & 307 & 36 & $11,7 \%$ & $88,3 \%$ \\
\hline
\end{tabular}

Table 2: Results of our method on real road images

Annexes, show some results. Limit of method are shown for cracks too thin and not oriented in one of the selected direction. Our method is disturbed by presence of spots in the texture, dark or white. We need to append at this detection some high level analysis to qualify spots detected. In Figure 8, we show a detection result on defect image of joint type. Our method can detect this defect type with "high" value of $d$ length ( in this example, $\mathrm{d}=28$ ).

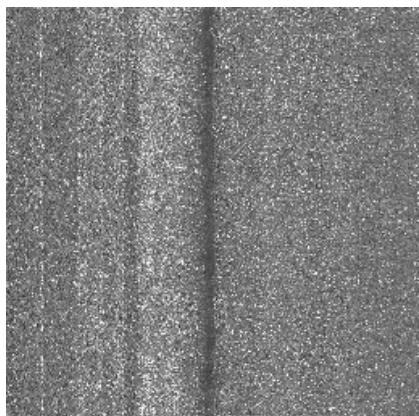

(a)

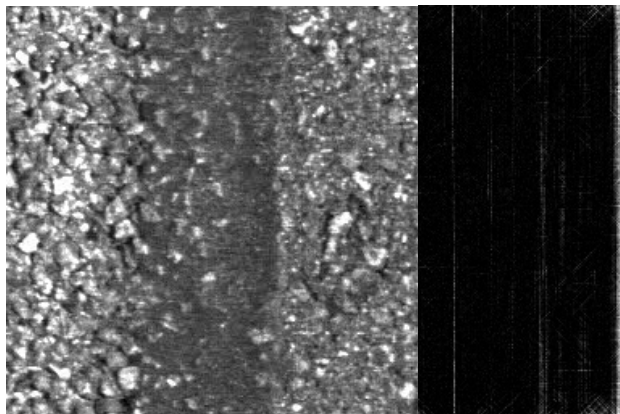

(b)

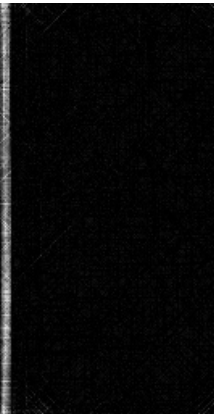

(c)

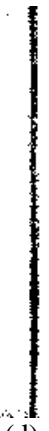

(d)

Fig. 8. Result of our method on image with joint type defect. Image (a), zoom (b), mahalanobis distance image (c), crack detection (d)

\section{Conclusion}

In this paper, we introduced a method for crack detection on road surface images. A measure of Conditional Texture Anisotropy (CTA) is used to characterize pixels on inspected images. Each pixel is segmented into "crack pixel class" or "crack-free pixel class" based on the CTA value.
Unlike other methods that only considered defect pixel as a local minimum in their segmentation step, our method transforms pixel from image space to CTA space using different parameters of features: extremum, continuity, homogeneity and dominant orientation.

Four traditional orientations $\left(0^{\circ}, 45^{\circ}, 90^{\circ}\right.$ and $\left.135^{\circ}\right)$ were used to calculate CTA in our first implementation, because of the rapidity in finding neighbor pixels without 
doing any interpolation $\left(0^{\circ}, 45^{\circ}, 90^{\circ}\right.$ and $135^{\circ}$ correspond to 4 directions in 8-connex grid). By this way, the method produces good results on cracks detection having these orientations (transversal crack, longitudinal crack).

We compare results with other methods in literature (threshold, wavelet transforms) and we show the efficiency of our method.

We are prospecting to improve pertinence of our distance to assess the probability of pixel to be of free defect type. Some new features should be added. We are adjusting a fuzzy approach which seems to perform good results. To avoid limitation of the "arbitrary" orientations used, we are developing a more generic algorithm. All these evolution should be included in a global system which use high level knowledge of road defects.

\section{References}

[1] Nguyen, TS., et al. Detection of Defects in Road Surface by a Vision System. in The 14th IEEE Mediterranean Electro-technical Conference. 2008. Ajaccio, France.

[2] Subirats, P., et al., Automation of Pavement Surface Crack Detection using the Continuous Wavelet Transform, in Image Processing, 2006 IEEE International Conference on. Oct. 2006: Atlanta, GA.

[3] Oliveira H., Lobato Correia P., Identifying and retrieving distress images from road pavement surveys, ICIP 2008 (57-60).

[4] Kumar, A., Grantham, and K.H. Pang, Defect detection in textured materials using optimized filters. Systems, Man and Cybernetics, Part B, IEEE Transactions on, Oct 2002. Vol.32: p. 553- 570.

[5] Chambon, S., J. Dumoulin, and P. Subirats, Introduction of a wavelet transform based on 2D matched filter in a Markov Random Field for fine structure extraction: Application on road crack detection, in IS $\backslash \& T / S P I E$ Conference on Image Processing: Machine Vision Applications II, San Jose, United-States. 2009.

[6] Cheng, H.D., et al., Novel approach to pavement cracking detection based on fuzzy set theory. Journal of Computing In civil Enginering, 1999. 13(4): p. 270-280.

[7] Chou J., W. O'Neill, and H.D. Cheng, Pavement distress evaluation using fuzzy logic and moment invariants. Transportation Research Record, 1995. 1505: p. 39-46.
[8] Chua, K.M. and L. Xu, Simple procedure for identifying pavement distresses from video images. Journal of transportation engineering, 1994. 120: p. 412431.

[9] Nallamothu, S. and K. Wang, Experimenting with recognition accelerator for pavement distress identification, in Transport Research Record. 1996. p. 130-135.

[10] Kalikiri, V.K., N.W. Garrick, and L.E.K. Achenie, Image-processing methods for automated distress evaluation. Transportation research record 1994. 1435: p. $45-51$.

[11] Tomita, F. and S. Tsuji, Computer analysis of visual textures. 1990: Kluwer Academic Publishers.

[12] Rasse, C., et al., Application of image analysis to the identification and rating of road surface distress. Bearing Capacity of Roads, Railways and Airfields, 2002. 6: p. 6168.

[13] Meignen, D., Méthodes d'imagerie: Premiers résultats, in Technical Report, Central Laboratory for Roads and Bridges 2003.

[14]Liu, Fanfan Xu, Guoai Yang, Yixian Niu, Xinxin Pan, Yuli, Novel Approach to Pavement Cracking Automatic Detection Based on Segment Extending, International Symposium on Knowledge Acquisition and Modeling, 2008. KAM'08.

[15] Li, Q. and X. Liu, Novel Approach to Pavement Image Segmentation Based on Neighboring Difference Histogram Method, in Proc. Congress on Image and Signal Processing CISP '08. 2008. p. 792--796.

[16] Delagnes, P., Extraction d'inhomogénéités dans les images texturées fixes et animées. 1996, Université de Nantes.

[17] Vectra's AMAC® specifications, Available from: http://www.vectra.fr/AMAC.pdf.

[18]CSIRO Manufacturing Science \& Technology, RoadCrack Specifications, Available from: http://www.cmis.csiro.au/iap/RecentProjects/roadcrack.ht $\mathrm{m}$.

[19] Roadware Group Inc, Wisecrax data sheet, Available from:

http://www.roadware.com/_lib/pdf/datasheet.wisecrax.pdf [20]Roli, F., Measure of texture anisotropy for crack detection on textured surfaces. Electronics Letters, 1996. vol. 32(no14): p. 1274-1275. 


\section{Some good results}

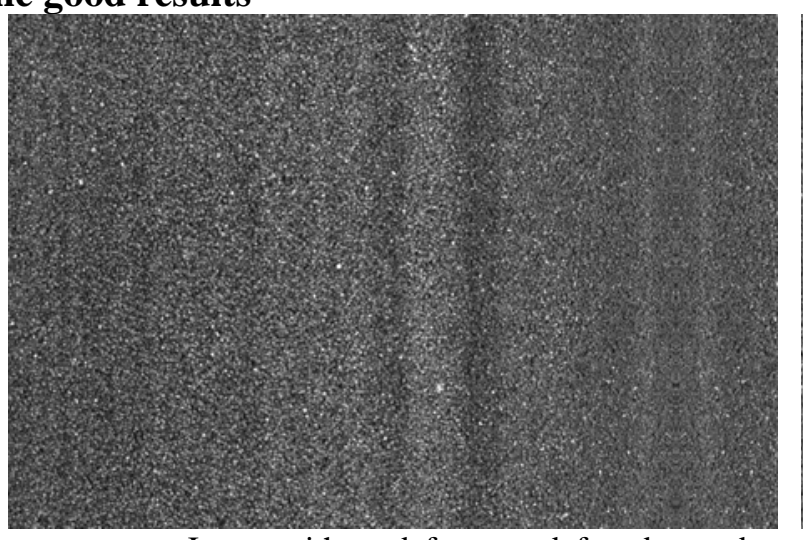

Image without defect : no defect detected

\section{Some fault detection}
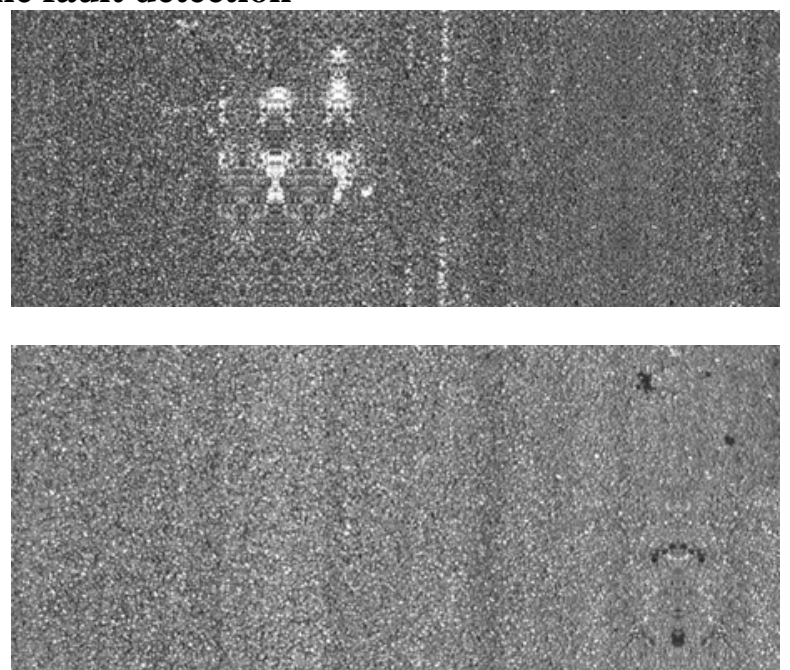

Image without defect : some defects detected

\section{Some defect not detected}
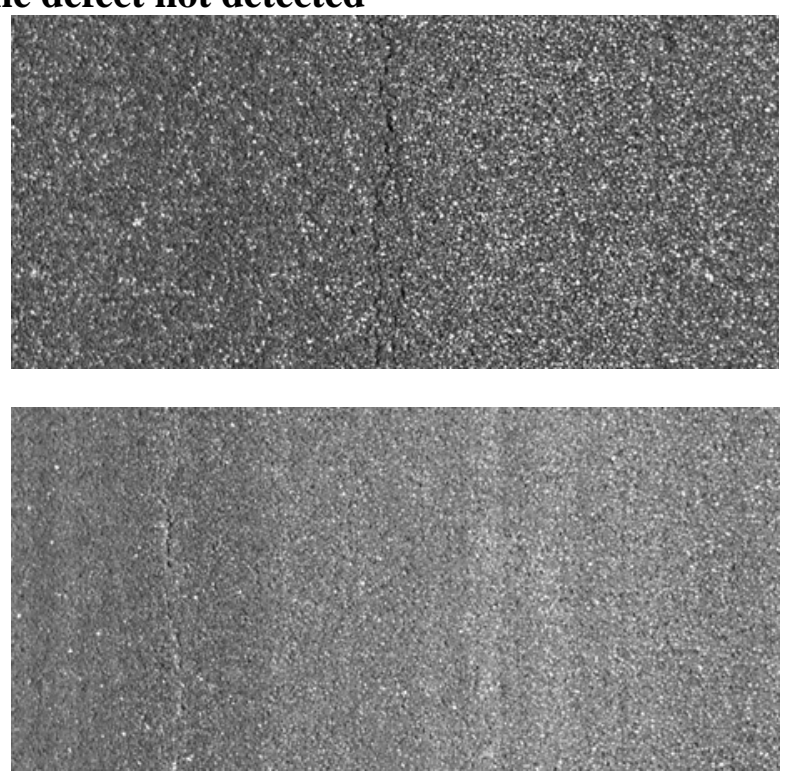

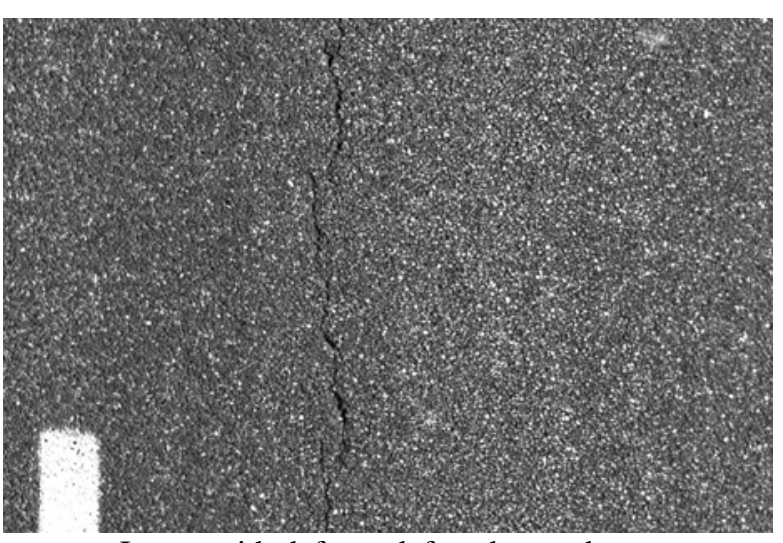

Image with defect : defect detected
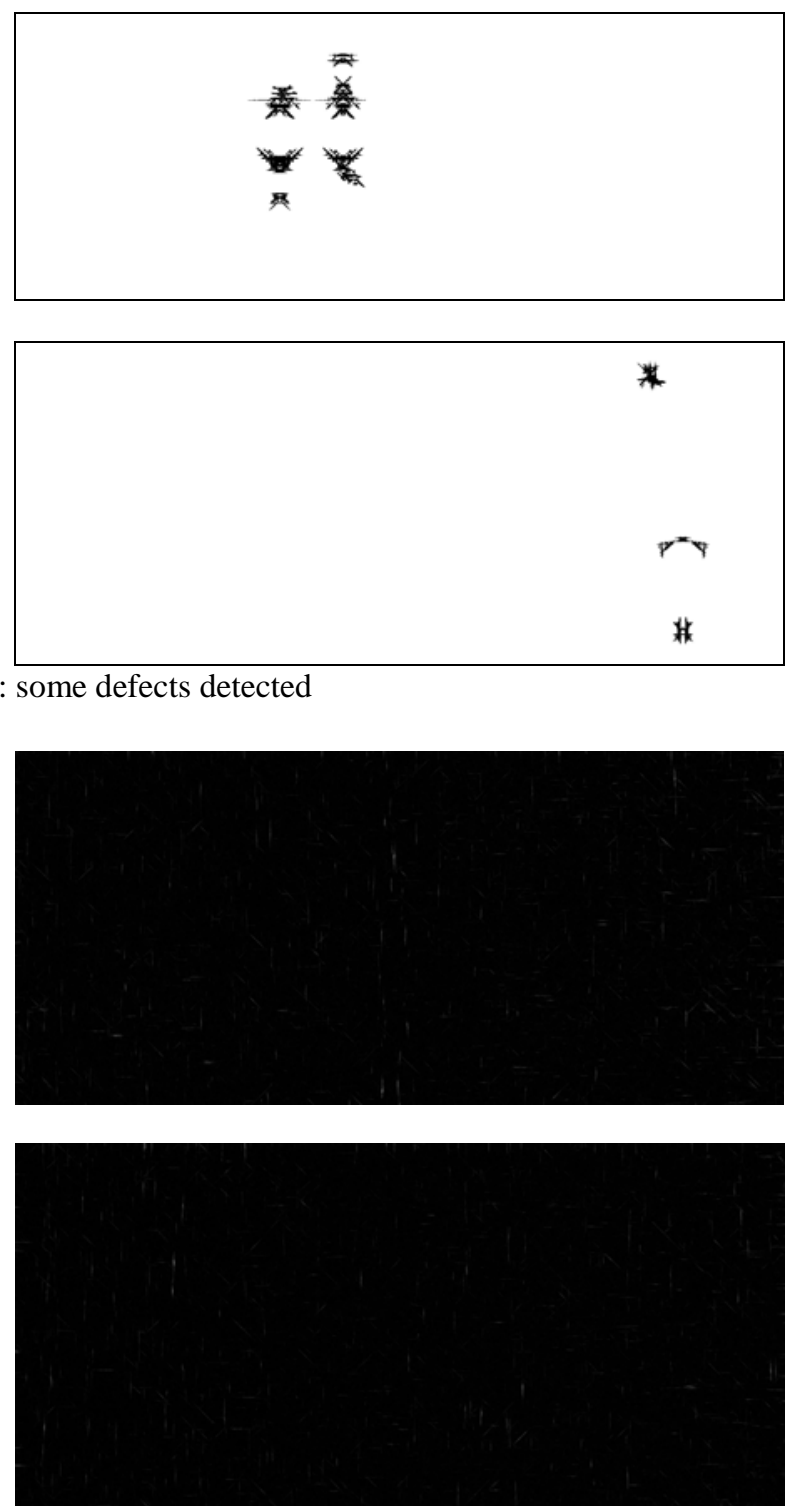

Image with defect : no detection 